

\section{La historia y los retos del desarrollo latinoamericano}

José Antonio Ocampo

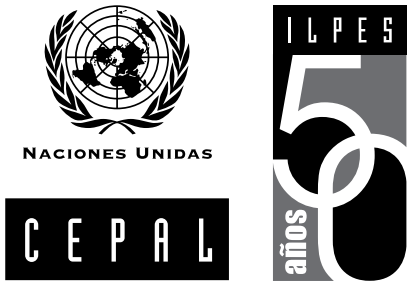

Comisión Económica para Amércia Latina y el Caribe (CEPAL) Santiago de Chile, noviembre de 2012 


\author{
Alicia Bárcena \\ Secretaria Ejecutiva \\ Antonio Prado \\ Secretario Ejecutivo Adjunto \\ Jorge Máttar \\ Director del \\ Instituto Latinoamericano y del Caribe de \\ Planificación Económica y Social (ILPES) \\ Ricardo Pérez \\ Director de la \\ División de Documentos y Publicaciones
}

Diseño de portada: Mario Tapia Rey

Publicación de las Naciones Unidas

LC/L.3546

LC/IP/L.322

Copyright (C) Naciones Unidas, diciembre de 2012. Todos los derechos reservados

Impreso en Naciones Unidas, Santiago de Chile • 2012-833

La autorización para reproducir total o parcialmente esta obra debe solicitarse al Secretario de la Junta de Publicaciones, Sede de las Naciones Unidas, Nueva York, N.Y. 10017, Estados Unidos. Los Estados miembros y sus instituciones gubernamentales pueden reproducir esta obra sin autorización previa. Sólo se les solicita que mencionen la fuente e informen a las Naciones Unidas de tal reproducción. 


\section{Índice}

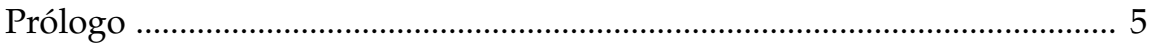

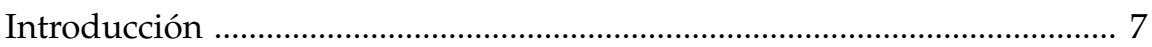

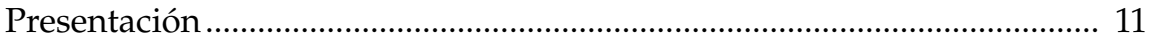

A. La herencia histórica ..................................................................... 13

B. ¿Qué mundo nos espera? ......................................................................... 23

C. Repensar la agenda del desarrollo ............................................................. 29

\section{Cuadros}

Cuadro 1 América Latina: crecimiento y volatilidad del PIB, 1950-1980 y 1990-2011 .......................................................... 17

\section{Gráficos}

Gráfico 1 América Latina: tasas decenales de crecimiento económico, 1900-2010 ........................................................... 14

Gráfico 2 América Latina: deuda externa, 1998-2011 ......................... 25

Gráfico 3 Mundo: crecimiento del comercio y del PIB, 1950-2012 ..... 26

Gráfico 4 América Latina: composición de las exportaciones, 1981-2010 



\section{Prólogo}

En épocas de elevada incertidumbre económica mundial, la reflexión sobre los dilemas y las oportunidades y la introspección acerca de los factores de nuestra historia que condicionan la concepción del futuro se tornan aún más relevantes.

Esta razón nos motiva a publicar la ponencia de José Antonio Ocampo, ex Secretario Ejecutivo de la Comisión Económica para América Latina y el Caribe (CEPAL) y actual profesor de la Universidad de Columbia, presentada en el seminario conmemorativo del quincuagésimo aniversario del Instituto Latinoamericano y del Caribe de Planificación Económica y Social (ILPES), realizado en julio de 2012.

El ILPES nació como una iniciativa regional ante las demandas políticas de planes y programas gubernamentales para fomentar el desarrollo económico. Raúl Prebisch supo combinar voluntades entre países de dentro y fuera de la región, para atender a la necesidad de profesionalizar y formalizar la planificación del desarrollo en América Latina y el Caribe.

Desde entonces, el Instituto ha cumplido con fidelidad la misión encomendada. Por supuesto, sufrió cuando la planificación pública fue menoscabada -innecesaria y equivocadamente - para desencadenar las fuerzas del mercado que por sí solas producirían el desarrollo. Pero mantuvo su línea y actualizó sus competencias para encontrarse hoy con un contexto político, económico y social que le abre nuevos espacios, dado el renovado interés en la planificación y el papel del Estado.

La CEPAL está proponiendo a sus Estados miembros un camino concreto hacia el crecimiento con igualdad y sostenibilidad ambiental: el cambio estructural, que rompe con el paradigma económico imperante en las últimas tres décadas. 
Para nosotros, hablar de igualdad implica difundir, a lo largo de la estructura productiva y el tejido social, el desarrollo de capacidades, el progreso técnico, oportunidades laborales plenas y el acceso universal a la protección social. El empleo con derechos es la llave maestra para superar la desigualdad y cerrar brechas con una mirada transversal en cuanto a equidad étnica, racial y de género.

En esencia, el cambio estructural para la igualdad que planteamos implica realizar transformaciones cualitativas en la estructura productiva de los países de la región, con el fin de fortalecer sectores y actividades intensivos en conocimiento y de rápido crecimiento de la demanda interna y externa, y obtener ganancias en productividad con más y mejores empleos.

Sostenemos que la clave está en coordinar las políticas macroeconómicas de corto plazo, el manejo del ciclo económico y la inversión con las políticas industriales de largo plazo y las políticas sociales redistributivas.

La sostenibilidad ambiental es uno de los ejes más importantes del cambio estructural y por eso es fundamental apropiarse de los nuevos paradigmas de la revolución tecnológica en la producción y establecer un patrón de diversificación sectorial que esté en sintonía con la sostenibilidad.

Proponemos un Estado proactivo que estimule las sinergias entre las políticas macroeconómicas y las políticas industriales y sociales. Se trata de apoyar a las pequeñas y medianas empresas, dar un salto en investigación y desarrollo y aprovechar las nuevas tecnologías para producir de forma limpia. Se trata de fortalecer la educación y la capacitación.

En nuestros días, la planificación entraña construir una visión de Estado de largo plazo, en estrecha coordinación con la sociedad civil y el sector privado organizado, como un esfuerzo colectivo y nacional que se articula en torno a la compleja pero fundamental trilogía Estado-mercado-sociedad.

Hoy la planificación pública está orientada a contener y alinear las fuerzas del mercado para que contribuyan a los objetivos de la sociedad en cuanto a desarrollo, igualdad y cambio estructural, con un Estado actuante y coordinador de estos esfuerzos de largo aliento.

Como señala Ocampo, la planificación, en un contexto de alta incertidumbre, aporta y contribuye a elaborar una visión y una orientación estratégicas.

\author{
Alicia Bárcena \\ Secretaria Ejecutiva \\ Comisión Económica para \\ América Latina y el Caribe (CEPAL)
}




\section{Introducción}

Con motivo de la celebración del quincuagésimo aniversario de la fundación del Instituto Latinoamericano y del Caribe de Planificación Económica y Social (ILPES), presentamos a los lectores la ponencia de José Antonio Ocampo, ex Director Nacional de Planificación de Colombia, ex Secretario Ejecutivo de la Comisión Económica para América Latina y el Caribe (CEPAL) y actual profesor de la Universidad de Columbia.

José Antonio Ocampo se refiere a la historia del desarrollo latinoamericano y caribeño, que constituye la raíz propia del ILPES, en su carácter de promotor y difusor del pensamiento y las ideas de la CEPAL, así como de la formación de miles de cuadros técnicos para la administración pública.

Cabe recordar que ya en los años cincuenta la CEPAL desarrollaba actividades para fortalecer las capacidades de gestión en los jóvenes gobiernos latinoamericanos. Raúl Prebisch, Secretario Ejecutivo de la CEPAL en ese momento, interpretó muy bien la creciente y diversificada demanda en estos ámbitos e impulsó la creación de una entidad que se hiciera cargo de dar respuestas de forma orgánica, lo que dio origen al ILPES.

La inauguración del curso de capacitación en desarrollo económico en Santiago, que tuvo lugar el 2 de julio de 1962, marca el nacimiento del Instituto. El curso puede considerarse la continuación del programa que la CEPAL venía ejecutando desde una década antes con el fin de apoyar a los gobiernos en el campo de la planificación y la gestión pública, mediante la prestación de servicios de capacitación, asesoría e investigación orientados a contribuir a los esfuerzos nacionales y subnacionales para mejorar la calidad de las políticas públicas y fortalecer el desarrollo institucional. 
Pero 1962 no fue un año cualquiera. Además de la fundación del ILPES, puede decirse que en ese año se inicia la planificación en América Latina, al crearse ministerios $u$ organismos encargados de esa labor en países como la Argentina, el Brasil, Colombia, Costa Rica, Cuba, El Salvador, México, el Paraguay y Venezuela (República Bolivariana de). En 1961 se firmó la Carta de Punta del Este, que dio origen a la Alianza para el Progreso, hito fundamental para el nacimiento de la planificación y punto de partida del acuerdo continental para fomentar el desarrollo estabilizador e incluyente, la industrialización y la cooperación e integración regionales (prioridades que siguen vigentes), con un Estado que se legitimaba como líder articulador de un proceso de dos décadas de crecimiento cercano al $6 \%$ en promedio en la región (un 5,5\% en los años sesenta y un 5,8\% en los años setenta).

El Instituto ha desarrollado su labor ininterrumpidamente a lo largo de cinco décadas, manteniendo las grandes líneas y el espíritu que le dieron origen, acompañando a los gobiernos en la construcción de capacidades institucionales y humanas, adaptándose a las necesidades cambiantes en función del desarrollo de los países, respondiendo a demandas emergentes en los diferentes ámbitos de la planificación y proveyendo un espacio de aprendizaje, reflexión, análisis y propuestas sobre los problemas del desarrollo latinoamericano y caribeño. Por las aulas del ILPES han pasado miles de alumnos que han llevado a los países la huella del pensamiento y quehacer de la CEPAL, muchos de los cuales han desempeñado y desempeñan hoy cargos de alta responsabilidad en sus gobiernos y otras entidades. El ILPES es, fue y será un espacio para el aprendizaje, la reflexión y el análisis de la planificación y el desarrollo latinoamericano y caribeño, abierto a todo el mundo.

La conmemoración del quincuagésimo aniversario de la fundación del Instituto, además de un orgullo, constituye un desafío y la renovación de un compromiso con América Latina y el Caribe. La región y el mundo han cambiado en estas cinco décadas; los países han avanzado en su desarrollo, pero se mantienen asignaturas pendientes que convocan a la CEPAL y al ILPES; los países reflexionan y discuten sobre el legado del esquema de desarrollo seguido en las dos últimas décadas, sobre la fórmula para reducir las desigualdades y sobre lo que parece ser un renovado interés por la planificación y las políticas para el desarrollo incluyentes, centradas en objetivos de igualdad en sus diferentes dimensiones, como la territorial, que tanto nos ocupa en el Instituto, pues es en ese espacio donde se manifiestan, se exacerban y se intentan resolver las desigualdades de ingreso, productividad y acceso a servicios como la educación y la salud.

El ILPES dialoga y colabora con los países sobre este nuevo enfoque y concepto de planificación, que responde a los organismos nacionales de 
desarrollo, cuya construcción y ejecución son, a diferencia del pasado, cada vez más participativas, como reflejo del avance democrático de la región. El Instituto apunta a ser una entidad regional de excelencia en la generación y difusión de conocimientos y propuestas sobre el papel del Estado en los procesos de desarrollo y en el mejoramiento de la planificación y la gestión pública.

Compartimos la alegría por los 50 años de la institución, rindiendo tributo y homenaje a los hombres y mujeres que han servido a la región, con entusiasmo, profesionalismo y dedicación, así como a quienes han acompañado esta labor desde la CEPAL y otros organismos como expertos y consultores, y desde los gobiernos, socios fundamentales de nuestro trabajo.

A quienes tenemos el honor y la ocasión histórica de pertenecer al ILPES en sus primeros 50 años, nos corresponde expresar y, a la vez, ser parte del reconocimiento que el Instituto merece, así como ratificar el compromiso con el desarrollo de la región. 



\section{Presentación}

José Antonio Ocampo*

La celebración de los 50 años del Instituto Latinoamericano y del Caribe de Planificación Económica y Social (ILPES) es motivo de inmenso orgullo. A lo largo de medio siglo, el ILPES ha contribuido a la formación de múltiples cuadros de profesionales de la región en áreas de la gestión y las políticas públicas, así como a la investigación en temas de planificación, finanzas públicas y desarrollo regional. Por lo tanto, en nombre de su actual director, Jorge Máttar, quiero felicitar a todos los directores y funcionarios que han contribuido al medio siglo de éxito de esta honrosa rama de nuestra querida Comisión Económica para América Latina y el Caribe (CEPAL).

Considero que esta ocasión es propicia para analizar la historia del desarrollo latinoamericano y, a partir de ella, reflexionar sobre los retos y opciones para la región. Mi punto de partida serán las consideraciones vertidas en una reciente historia económica de América Latina, escrita en conjunto con Luis Bértola, El desarrollo económico de

* Profesor de la Escuela de Asuntos Internacionales y Públicos y miembro del Comité de Asuntos Globales de la Universidad de Columbia. Previamente, Secretario General Adjunto para Asuntos Económicos y Sociales de las Naciones Unidas, Secretario Ejecutivo de la Comisión Económica para América Latina y el Caribe (CEPAL) y Ministro de Hacienda de Colombia. Esta es una versión escrita de la presentación que hizo el autor en el acto de celebración del quincuagésimo aniversario del ILPES el 2 de julio de 2012. Por su naturaleza, no sigue el formato académico y no incluye, en particular, referencias bibliográficas. 
América Latina desde la independencia ${ }^{1}$. Sobre esa base, haré algunas reflexiones sobre la coyuntura actual y lo que ella nos revela acerca del mundo futuro y expondré algunas breves conclusiones sobre la agenda del desarrollo latinoamericano.

$1 \quad$ Esta obra será publicada en 2012 por Oxford University Press (en inglés) y en 2013 por el Fondo de Cultura Económica. La Secretaría General Iberoamericana (SEGIB) publicó una versión previa en 2010 bajo el título Desarrollo, vaivenes y desigualdad: Una historia económica de América Latina desde la independencia. En la primera sección se utilizan ampliamente los análisis de esta obra, especialmente del capítulo 6. 


\section{A. La herencia histórica}

Una mirada sucinta a las tendencias de largo plazo nos indica que América Latina logró su mayor crecimiento económico durante el período de industrialización liderada por el Estado². Esto se refleja en el gráfico 1, en el que se muestran promedios móviles del crecimiento observado durante determinadas décadas. Como se puede apreciar, el ritmo de crecimiento, de más del $5 \%$ y el $6 \%$ anual hacia el final del período, solo se alcanzó fugazmente durante la etapa previa de desarrollo de las exportaciones de productos primarios (en particular, en la década que antecedió a la Primera Guerra Mundial y en los años veinte). Por el contrario, después de las reformas económicas, uno de cuyos objetivos fue precisamente acelerar el crecimiento económico, los ritmos alcanzados han sido muy inferiores y apenas se han acercado en los últimos diez años al $4 \%$ anual. Debido a las tendencias demográficas, el crecimiento del PIB per cápita se vio deprimido durante la industrialización dirigida por el Estado debido a la explosión demográfica $y$, por el contrario, se ha visto beneficiado en épocas recientes por el bono demográfico (la reducción de las tasas de dependencia).

Preferimos este término al de sustitución de importaciones, ya que este último no capta más que una característica de ese período de desarrollo y no necesariamente la más importante. En la terminología de la CEPAL también se ha denominado "desarrollo hacia adentro". 


\section{Gráfico 1}

AMÉRICA LATINA: TASAS DECENALES DE CRECIMIENTO ECONÓMICO, 1900-2010

(En porcentajes, sobre la base del crecimiento anual medio)

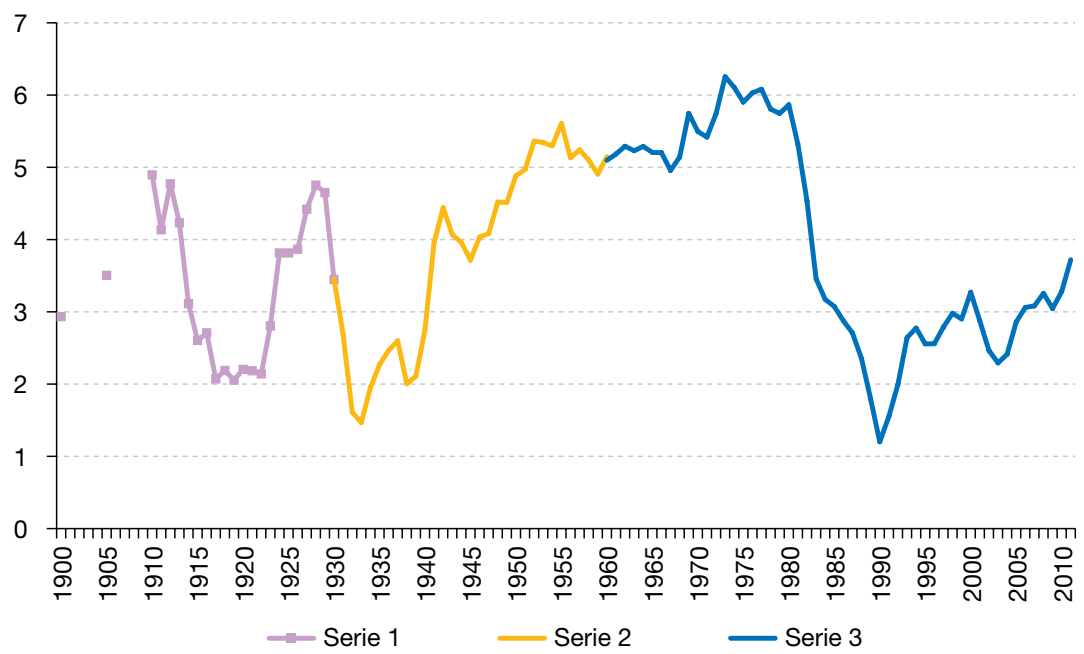

Fuente: Luis Bértola y José Antonio Ocampo, El desarrollo económico de América Latina desde la independencia, México, D.F., Fondo de Cultura Económica, 2013, en prensa.

Nota: La serie 1 incluye a la Argentina, el Brasil, Chile, Colombia, Cuba, el Ecuador, México, el Perú, el Uruguay y Venezuela (República Bolivariana de); en las dos primeras observaciones se excluyen Cuba y el Ecuador. La serie 2 incluye a todos los países, excepto Bolivia (Estado Plurinacional de), Panamá, el Paraguay y la República Dominicana. La serie 3 incluye a todos los países, excepto Cuba.

Durante la fase de desarrollo de las exportaciones de productos primarios, América Latina fue, con Europa central y meridional, una de las regiones de la periferia de la economía mundial que lograron insertarse en forma más temprana al proceso de crecimiento económico, lo que la convirtió en una especie de "clase media" del mundo. Como resultado, entre 1870 y 1929, la participación de América Latina en el PIB mundial aumentó del 2,6\% al 5,2\% y la región creció a un ritmo per cápita superior al promedio mundial ${ }^{3}$. Durante la industrialización dirigida por el Estado, la economía latinoamericana siguió creciendo algo más que el promedio y su participación en la producción mundial continuó incrementándose, hasta alcanzar un 9,5\% en 1980. Sin embargo, en ninguna etapa logró recortar más que marginalmente la distancia que ya la separaba en 1870 del mundo desarrollado, y durante la "edad de oro" del mundo industrializado, entre 1950 y 1973, quedó rezagada en relación con Europa occidental. Durante la década perdida de 1980, la participación en el PIB mundial se redujo al 8,0\% y siguió

3 Véase el cuadro 1.1 en Bértola y Ocampo (2013). 
descendiendo lentamente desde entonces. De esta manera, la fase más reciente de desarrollo ha sido de retroceso relativo, ahora en relación con el promedio mundial y, sobre todo, con los países en desarrollo de Asia. Dado que la primera y la tercera de estas etapas coinciden con los procesos modernos de globalización, puede decirse que América Latina obtuvo ganancias durante la primera globalización, pero no se ha podido beneficiar plenamente de la segunda, pese a su relativo buen desempeño exportador, a la capacidad de atraer capitales y a que las grandes empresas de la región (las llamadas translatinas) han jugado un papel exitoso en la región y a nivel mundial.

El desarrollo se difundió en forma desigual dentro de la región. Esto fue particularmente cierto durante la etapa de desarrollo de las exportaciones primarias. En efecto, ya antes de la Primera Guerra Mundial los países del Cono Sur y, en menor medida, Cuba, habían logrado ampliar sus ventajas en relación con el resto de la región. Desde entonces se inició un proceso de convergencia de los niveles de desarrollo regional, producto tanto del rezago que comenzaron a experimentar los líderes como del éxito tardío de otros países, sobre todo los dos más grandes (Brasil y México) pero también algunos medianos (Colombia y Venezuela [República Bolivariana de]) y pequeños (Costa Rica, Ecuador y Panamá, en particular). No obstante, algunos países quedaron rezagados (Bolivia [Estado Plurinacional de]) y Nicaragua son los casos más relevantes). Este proceso de convergencia regional se detuvo durante la década perdida de los años ochenta y en las últimas décadas se reinició una tendencia divergente.

El proceso de crecimiento económico de determinados países muestra otro patrón destacado: la tendencia de los países latinoamericanos a experimentar prolongadas fases de rápido crecimiento, que reducen por un tiempo la brecha de ingresos que los separa de los países industrializados, pero que son sucedidas por grandes retrocesos relativos durante otro período también prolongado, un fenómeno que se puede denominar de "convergencias truncadas". Cuba fue el caso más temprano de este patrón: después de haber tenido uno de los grandes éxitos exportadores del siglo XIX y comienzos del XX, experimentó un virtual estancamiento del ingreso por habitante desde mediados de la década de 1910. La historia del Cono Sur es similar: se observa un gran avance hasta la Primera Guerra Mundial y un retroceso relativo posterior. Esto fue particularmente notorio en la Argentina, uno de los grandes éxitos a nivel mundial durante la primera globalización. Le sigue en la lista Venezuela (República Bolivariana de), el país con mayor ritmo de crecimiento de la región entre las décadas de 1920 y 1960 - gracias a su despegue petrolero y la capacidad de aprovechar parte de sus beneficios-, período sucedido por un marcado retroceso relativo 
posterior. El Brasil y México, los grandes éxitos de la industrialización dirigida por el Estado, siguieron esa ruta poco después. Puede decirse quizás que la ausencia de "milagros", pero también de grandes crisis y, por ello, la capacidad de tener un desarrollo pausado pero estable, es el curioso secreto de Colombia.

A ello se une la elevada vulnerabilidad externa de las economías latinoamericanas y la volatilidad del crecimiento económico que ha resultado de ella. El factor que ha tenido efectos a lo largo de la historia económica de la región ha sido la dependencia de los productos básicos, sujetos a una alta volatilidad de los precios, que además se ha agudizado en algunos períodos, en particular entre la Primera Guerra Mundial y la Gran Depresión y desde mediados de los años setenta. A ello se agrega la volatilidad aún más acentuada que ha resultado del acceso irregular y fuertemente procíclico al financiamiento externo, que ha generado algunos de los ciclos más pronunciados: el auge de la segunda mitad de los años veinte, sucedido por la dura contracción y la moratoria virtualmente generalizada de la deuda externa de los años treinta; el auge de la segunda mitad de la década de 1970, seguido por la década perdida de 1980; el crecimiento económico de 1991-1997, sucedido por la interrupción súbita del financiamiento externo a partir de la crisis asiática de 1997 y la rusa de 1998, que duraría media década, y el auge que antecedió a la crisis financiera mundial de 2008 y la fuerte contracción que tuvo lugar entonces, que resultó mucho más corta que las anteriores, ya que se produjo una nueva expansión del financiamiento externo a partir de mediados de 2009.

Una de las dimensiones de la vulnerabilidad externa ha sido la marcada volatilidad del crecimiento económico que ha experimentado la región durante el período de reformas de mercado. Ese comportamiento se refleja con claridad al comparar la inestabilidad de los ritmos de crecimiento del período 1990-2011 con los que habían caracterizado al período 1950-1980 (véase el cuadro 1). Esa mayor volatilidad refleja tanto los vaivenes del financiamiento externo y de los términos de intercambio generados por los ciclos de precios de los productos básicos, como la tendencia de la política macroeconómica a seguir un patrón procíclico, que solo ha comenzado a superarse parcialmente en épocas recientes. Uno de los resultados de ello es que, si bien ha habido avances en materia de estabilidad de precios y en evitar las crisis financieras, se ha logrado menos en otra dimensión de la estabilidad macroeconómica, que de hecho se ignora con frecuencia cuando se utiliza este término: la estabilidad de los ritmos de crecimiento económico. 
Cuadro 1

AMÉRICA LATINA: CRECIMIENTO Y VOLATILIDAD DEL PIB, 1950-1980 Y 1990-2011 a

(En porcentajes)

\begin{tabular}{lccc}
\hline $\begin{array}{l}\text { Promedio } \\
\text { ponderado }\end{array}$ & $\begin{array}{c}\text { Crecimiento } \\
\text { medio }\end{array}$ & $\begin{array}{c}\text { Desviación } \\
\text { estándar }\end{array}$ & $\begin{array}{c}\text { Coeficiente } \\
\text { de variación }\end{array}$ \\
\hline $1950-1980$ & 5,5 & 1,7 & 0,31 \\
$1990-2011$ & 3,3 & 2,4 & 0,71 \\
\hline Promedio simple & & & 0,21 \\
\hline $1950-1980$ & 5,0 & 1,1 & 0,50 \\
$1990-2011$ & 4,0 & 2,0 & \\
\hline
\end{tabular}

Fuente: Elaboración propia sobre la base de datos de la Comisión Económica para América Latina y el Caribe (CEPAL).

a No se incluye a Cuba.

Las consideraciones previas dejan claro que la "leyenda negra" que se ha tejido sobre la fase de industrialización dirigida por el Estado no está basada en una observación detenida de la historia. Este período de desarrollo no solo ha sido la etapa de crecimiento más rápido durante un período prolongado, sino también de crecimiento estable y, como veremos, de avance social. Fue incluso, al menos desde mediados de los años sesenta (y antes, en el caso de las economías más pequeñas), una etapa de crecimiento y diversificación exportadora, cuando el modelo clásico de desarrollo hacia adentro dio paso a un modelo mixto que combinaba sustitución de importaciones con promoción de exportaciones e integración regional. Sin embargo, resultaría iluso retornar a un patrón de desarrollo de este tipo, cuyos orígenes históricos estuvieron estrechamente ligados al colapso de la primera globalización más que a cualquier otro factor. Por lo tanto, el retorno resultaría anacrónico en la segunda globalización que vivimos hoy.

Además, este modelo manifestó también deficiencias, en particular, su incapacidad para crear una base tecnológica sólida. Esa incapacidad tiene profundas raíces, ya que se remonta al atraso industrial experimentado durante la primera globalización, a los rezagos educativos acumulados y los retrasos aún mayores en la construcción de una base científico-tecnológica propia. A ello se agregó, desde mediados de la década de 1970, la reversión del proceso de industrialización, ya que tuvo lugar en una etapa todavía temprana del desarrollo, cuyo reflejo fue el freno de la tendencia ascendente de los niveles de productividad que habían experimentado la mayoría de las economías latinoamericanas hasta ese momento. 
En contra de las expectativas de los reformadores económicos, la mayor y en muchos sentidos exitosa integración de América Latina a la economía mundial, los procesos paralelos de liberalización económica que la acompañaron y las tendencias adversas de la productividad que se iniciaron a partir de la década perdida se mantuvieron hasta la primera mitad de la década de 2000. Este hecho no fue inconsistente con un buen desempeño de la productividad en las empresas líderes, sino más bien el resultado del creciente dualismo o heterogeneidad estructural que caracterizó la primera fase de las reformas: la coexistencia de empresas de talla mundial (entre ellas, las transnacionales presentes en la región y las translatinas) y un mundo creciente de pequeñas empresas y empleo informal. Por eso, la tendencia adversa de la productividad total de los factores solo se comenzó a revertir durante el auge de 2003-2007, cuando los indicadores de informalidad laboral comenzaron a descender en forma consistente.

Cabe agregar que hasta comienzos de los años setenta, la propensión a la inflación fue una característica casi exclusiva del Cono Sur y el Brasil y que la indisciplina fiscal solo se generalizó en la fase de abundancia de financiamiento externo, durante la segunda mitad de la década. Por ello, el desborde inflacionario fue más un efecto que una causa de la crisis de la deuda externa. Los logros en ambas materias son, por supuesto, un éxito del manejo macroeconómico de las dos últimas décadas. Ello ha representado un avance histórico neto para el Brasil y los países del Cono Sur y un retorno a lo que era típico hasta la década de 1960 para el resto de los países.

En materia social, los progresos vinieron con más rezago. El lastimoso estado de la educación a comienzos del siglo XX, incluso en los países que lideraron el desarrollo regional, es una muestra de ello. Los indicadores de desarrollo humano comenzaron a mejorar hacia la tercera década del siglo XX y tuvieron los mayores avances durante la industrialización dirigida por el Estado, en forma relativamente generalizada a lo largo y ancho de la región. Durante las dos últimas décadas del siglo XX mostraron un estancamiento en relación con el mundo industrializado, aunque con un continuado avance en materia educativa. La reducción de la brecha de desarrollo humano con los países desarrollados se reanudó con firmeza en la primera década del siglo XXI.

En materia de reducción de la pobreza, los pocos estudios existentes indican que los mayores avances del siglo $\mathrm{XX}$ tuvieron lugar nuevamente durante el período de industrialización dirigida por el Estado. Esta tendencia favorable fue sucedida no por una década, sino por un cuarto de siglo perdido en materia de reducción de la pobreza a partir de 1980. Uno de los hechos más notorios de épocas recientes ha sido la marcada 
reducción de los niveles de pobreza que se produjo entre 2003 y 2008, y coincidió con una mejoría de la distribución del ingreso en un conjunto amplio de países.

La historia de la desigualdad interna es compleja y diversa, y no sigue un patrón único. Por supuesto que sigue pesando hasta hoy la herencia colonial de sociedades altamente segmentadas económica y socialmente, pero su dinámica se vio afectada por otros factores, que han tenido una incidencia diversa en distintos países de la región. Los efectos adversos más generalizados en materia distributiva fueron el resultado de la primera globalización, la crisis de la deuda de la década de 1980 y la liberalización económica de fines del siglo XX. Para los países con alto excedente de mano de obra (esencialmente todos, excepto los del Cono Sur), la presión que estos generaron durante buena parte del siglo XX tuvo también efectos negativos en materia distributiva. A ello se ha sumado el sello adverso que han dejado muchas dictaduras militares.

Sin embargo, también ha habido fuerzas positivas. A la postre, la mayor ganancia en materia de igualdad social ha sido la abolición de la esclavitud, que ocurrió en forma muy tardía en algunos países (Brasil y Cuba) y la más lenta erosión de las formas serviles de trabajo rural que predominaban incluso a comienzos del siglo XX en el grueso de los países latinoamericanos y siguieron teniendo incidencia por mucho tiempo. La urbanización brindó oportunidades a poblaciones rurales que habían vivido bajo el signo de la fuerte segmentación social que caracterizaba a las sociedades rurales de la región. El avance tardío de la educación fue también una fuerza igualadora y quizás la fuente más importante de la mejora distributiva de comienzos del siglo XXI. No obstante, este avance ha sido incompleto, como se refleja en los rezagos y desigualdades en términos de la calidad de la educación a la que accede la mayoría de los latinoamericanos. En los países del Cono Sur, la gran migración europea tuvo a la larga efectos distributivos favorables, entre otras cosas, porque los inmigrantes trajeron consigo habilidades, conocimientos $\mathrm{y}$, muy especialmente, instituciones (entre las que se destaca el sindicalismo) que contribuyeron a difundir los beneficios de los procesos de desarrollo. Otros países han tenido giros institucionales hacia la equidad, muy notablemente los que tuvieron lugar en Costa Rica a mediados del siglo XX o en Cuba con su revolución.

La forma en que se combinaron estas fuerzas de diverso signo fue muy variada de un país a otro y no existe información para corroborar con plenitud sus efectos netos, pero es posible distinguir dos grandes ciclos. El primero tuvo una fase de deterioro hasta comienzos del siglo XX e incluso después en economías con excedente de mano de obra. A ella le sucedió una etapa de mejoría, que se dio en forma temprana (desde la década de 1920) 
en el Cono Sur - debido a los factores institucionales mencionados-, en forma más tardía (en las décadas de 1960 o 1970) en otros países (Colombia, Costa Rica, México y Venezuela (República Bolivariana de)), pero que no se produjo nunca en algunos (Brasil). El segundo ciclo se inició con una fase de deterioro que inauguraron nuevamente los países del Cono Sur, pero se generalizó a fines del siglo XX con la década perdida y las reformas de mercado. A ello le sucedió una fase de mejoría distributiva en la primera década del siglo XXI en dos terceras partes de los países de la región. A largo plazo, puede decirse que la desigualdad en la distribución del ingreso de América Latina, aparte de ser estructuralmente alta, es quizás más acentuada hoy que cuando se inició el proceso de rápido crecimiento económico, en las últimas décadas del siglo XIX.

La historia del desarrollo institucional, entendido como la organización del Estado y, más en general, la sociedad para la provisión de bienes públicos, muestra también una notoria diversidad regional, aún en países que comparten fronteras y procesos similares de desarrollo en otros sentidos, sin que exista ninguna explicación más que la trayectoria histórica de cada uno de ellos. Un elemento común, del que escapan solo un puñado de países (y solamente en forma parcial) es la ausencia de sincronía entre el liberalismo económico y el liberalismo político, que por fin logra afianzarse en las dos últimas décadas del siglo $\mathrm{XX}, \mathrm{y}$ aun así con retrocesos recientes. Otro elemento común ha sido la tendencia al rentismo, que ha recaído alternativamente sobre la dependencia de las rentas de los recursos naturales o de las que proporciona la relación privilegiada con el Estado.

En cuanto a la relación entre el Estado y el mercado, la etapa de industrialización dirigida por el primero representó el mayor avance en término de desarrollo de las actividades estatales, pero en muchos casos las instituciones correspondientes tenían antecedentes en la etapa previa de desarrollo de las exportaciones primarias. Los países de la región desarrollaron durante la etapa de industrialización un modelo de economía mixta similar al de Europa occidental, con pocas excepciones (Cuba y los experimentos socialistas temporales que tuvieron lugar en un puñado de otros países). Ese modelo fue mucho menos estatista en América Latina que en la mayoría de los países en desarrollo de Asia y África, un hecho que se ignora a menudo. Esta fue también la época clásica de la planificación.

Desde los años setenta en unos pocos países y desde mediados de la década de 1980 en el grueso de ellos, la liberalización económica produjo una importante redefinición de la relación entre Estado y mercado. Sin embargo, dicha redefinición permitió la sobrevivencia de empresas y bancos públicos en la mayoría de los países, y en todos se caracterizó por una ampliación significativa del gasto público social desde 1990. Hubo 
también un proceso de fortalecimiento de algunas entidades estatales, en especial de ministerios de hacienda y bancos centrales, pero también el retroceso o incluso la desaparición de las entidades de planificación y de las encargadas de las políticas de desarrollo productivo (tanto industrial como agrícola). Curiosamente, la recuperación del gasto social no estuvo acompañada en las últimas décadas de una tendencia similar del gasto en infraestructura, por lo cual la región acumuló un rezago considerable en ese campo. A su vez, la falta de atención aún mayor a la ciencia y la tecnología generó un rezago considerable en relación con los grupos de referencia relevantes (tanto en los países de Asia Oriental como en las economías desarrolladas con estructuras productivas en las que pesan los recursos naturales).

La frustración respecto de las reformas de mercado dio lugar a un retorno a visiones más positivas del papel del Estado. Este hecho se reflejó en un surgimiento de movimientos de izquierda de diferente índole, pero es un proceso que se dio aún en países con orientaciones de centro o incluso de derecha. En este sentido, América Latina lideró un proceso que abarca ahora a un conjunto más amplio de países del mundo. En todo caso, se trata de una tendencia diversa y con fallas notorias. Entre ellas se cuenta, en particular, la ausencia de una mayor atención a las políticas de desarrollo productivo. El Brasil es el único que ha montado una política ambiciosa en ese campo, seguido muy de lejos por unos pocos países. 



\section{B. ¿Qué mundo nos espera?}

La incertidumbre que rodea a la economía mundial es, sin duda, una de las más grandes de que tengamos memoria, solo comparable en términos económicos a la que rodeó a la Gran Depresión de los años treinta. El elemento central es la crisis financiera que ha afectado a los Estados Unidos y Europa occidental y que, por la importancia de las economías involucradas, se ha denominado crisis financiera mundial, pero que quizás se debería denominar más correctamente la crisis financiera del Atlántico Norte.

La característica más reciente ha sido la agudización de la crisis en Europa occidental, a medida que la crisis financiera ha comenzado a vincularse en forma compleja a la crisis fiscal de varios de los países de la zona del euro, amenazando la estabilidad de la unión monetaria. El compromiso de los países miembros puede evitar el colapso del euro, pero la lentitud de los procesos decisorios ha condenado ya a Europa a una década perdida y quizás más. La razón básica es la enorme asimetría de los procesos de ajuste en curso, ya que los ajustes fiscales y salariales recesivos que tienen lugar en los países de la periferia europea no tienen su contraparte en políticas fiscales y salariales expansivas en los países de esta zona con mayores fortalezas relativas. La resolución todavía incompleta de la crisis griega implica que subsiste el peligro de ruptura de la zona del euro, ya que generaría procesos de contagio masivo en otros países de la periferia europea que serían muy difíciles de manejar. Cabe agregar que un colapso del euro, la segunda moneda de reserva a nivel internacional, arrastraría sin duda a la economía mundial a una recesión igual o peor que la que tuvo lugar después de la quiebra del 
banco de inversión estadounidense Lehman Brothers en septiembre de 2008.

Entre tanto, el Banco Central Europeo (BCE) ha actuado eficazmente como proveedor de liquidez de los bancos, pero sus intervenciones en los mercados de bonos de deuda pública han sido intermitentes y la entidad no ha dado hasta ahora ninguna señal de tener la firme decisión de estabilizar dichos mercados. Con cierta razón, el BCE ha querido que la función de estabilizar los mercados de deuda pública recayera sobre los mecanismos de estabilidad creados por los países miembros, pero la magnitud de los recursos asignados a ellos es demasiado limitada para lograr este objetivo. A todo ello se agrega la lentitud de ciertos procesos, especialmente la instrumentación de la regulación y supervisión común de los sistemas bancarios (denominada unión bancaria) y la puesta en marcha de reglas fiscales más rigurosas (denominada incorrectamente unión fiscal, ya que no incluye otros elementos de una verdadera unión fiscal, en particular una redistribución significativa de recursos públicos entre sus miembros). A corto plazo, como se señaló, las medidas fiscales han acentuado la recesión.

En los Estados Unidos, la relativa debilidad del proceso de reactivación, asociado a los ajustes aún en curso de los balances del sector privado y especialmente de los hogares, se combina ahora con los enfrentamientos políticos agudos que están conduciendo a la indefinición de caminos razonables para manejar la trayectoria del déficit y la deuda pública. La amenaza inmediata es el llamado "precipicio fiscal" (fiscal cliff) que experimentará ese país a comienzos de 2013, cuando se combinarán los ajustes automáticos del gasto público decretados por el Congreso con el vencimiento de los beneficios tributarios del gobierno de Bush y de los otorgados en los últimos años a las contribuciones a la seguridad social. De acuerdo con la Oficina de Presupuesto del Congreso, ello daría lugar a un ajuste fiscal inmediato del 5,1\% del PIB que produciría una recesión en el primer semestre del próximo año.

En todo caso, e independientemente de esa coyuntura crítica y de la profunda división política que refleja, la economía estadounidense ha experimentado una reactivación menos errática que la europea. La política fiscal ha tenido una trayectoria anticíclica más razonable y, sobre todo, se ha combinado con un activismo de la Reserva Federal de los Estados Unidos, que se ha reflejado en una política monetaria expansionista que ha cumplido media década y promete continuar. Su reflejo en la reactivación del crédito ha sido lento, pero se ha manifestado ya en una recuperación del crédito a las empresas y al consumo, aunque no todavía del hipotecario. Ello indica también que, a diferencia de Europa, el sistema financiero parece haber alcanzado una situación relativamente estable. 
La conjunción de las debilidades de Europa occidental y, en menor medida, de los Estados Unidos, a los que podríamos agregar el Japón, implican que el centro de la economía mundial (para utilizar la famosa figura del creador del ILPES, Raúl Prebisch), no generará grandes impulsos dinámicos en el futuro inmediato e incluso mediato. El impacto sobre América Latina se dará por tres vías diferentes. La migración de mano de obra hacia el centro (Estados Unidos y España, en particular) dejó ser el factor favorable que fue durante las décadas anteriores, lo que ha tenido un impacto adverso para la región, en particular para el Ecuador y varios países pequeños y medianos de Centroamérica y el Caribe. Los flujos de capital se redujeron drásticamente después del colapso de Lehman Brothers, pero se recuperaron con rapidez y resultaron afectados solo en forma marginal por la crisis europea. Por eso, salvo que ocurra un colapso de la zona del euro, lo que generaría una crisis mundial que afectaría también a la región, tendrán un efecto mucho menos desfavorable que en el pasado. La razón de ello es la gran reducción del endeudamiento externo que experimentó América Latina durante la década pasada (véase el gráfico 2), que genera la correcta percepción de ser una región de bajo riesgo para los capitales internacionales, con la excepción de algunos países sujetos a una percepción de alto riesgo político. Por el contario, en algunos casos se han generado en años recientes flujos de capital que pueden resultar excesivos para la capacidad de absorción de algunos países.

\section{Gráfico 2}

AMÉRICA LATINA: DEUDA EXTERNA, 1998-2011

(En porcentajes del PIB, a tasas de cambio de 2000)

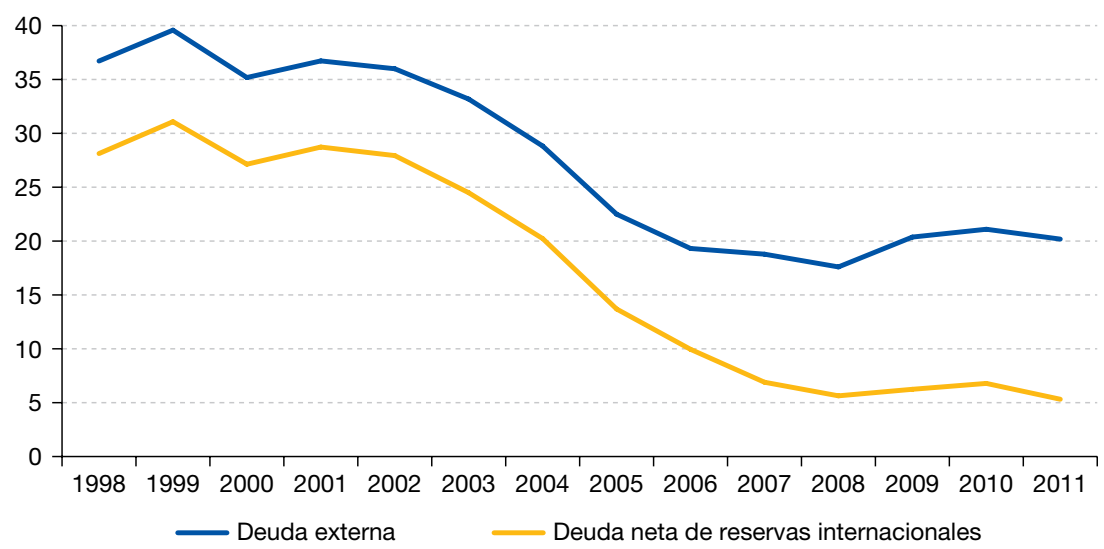

Fuente: Elaboración propia sobre la base de datos de la Comisión Económica para América Latina y el Caribe (CEPAL). 
Las principales consecuencias, y aquellas con efectos más generalizados en los países de la región, se producirán a través del comercio internacional. El problema principal es que el gran auge que experimentó el comercio internacional entre 1950 y 1974 y entre 1986 y 2007, ha llegado a su fin. Desde 2007 se ha producido una drástica desaceleración del ritmo de crecimiento de dicho comercio, no solo por la marcada reducción del ritmo de crecimiento de la economía mundial, sino también por la menor elasticidad entre el crecimiento del comercio y el del PIB mundial (un $1,5 \%$ en el período reciente en comparación con un 2,4\% durante el auge de 1986-2007). Esta situación es crítica porque los países latinoamericanos apostaron exclusivamente al desarrollo exportador siguiendo, además, una estrategia más bien pasiva, que otorgaba poco énfasis al contenido tecnológico de la canasta exportadora. Por lo tanto, parte del éxito de la aceleración del crecimiento exportador, apenas parcial, como vimos, en términos de impulsar el crecimiento económico y aumentar la productividad, se debe a condiciones del comercio internacional que no se presentarán en los próximos años.

Gráfico 3

MUNDO: CRECIMIENTO DEL COMERCIO Y DEL PIB, 1950-2012

(En porcentajes)

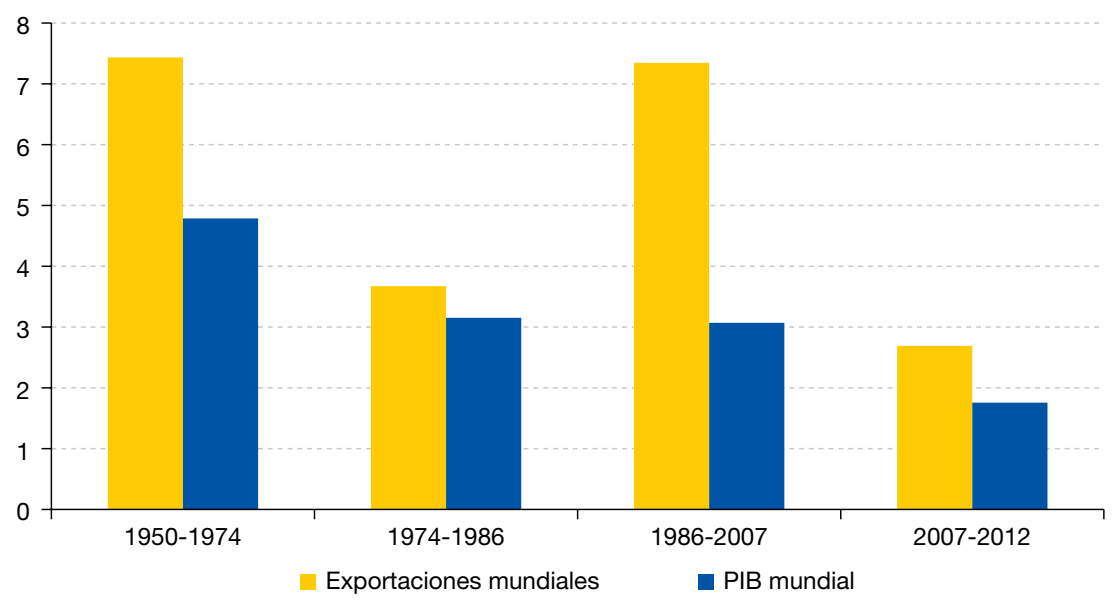

Fuente: Elaboración propia sobre la base de datos de las Naciones Unidas hasta 2007; y del Fondo Monetario Internacional para 2007-2012.

Nota: EI PIB se estima a precios de mercado.

Un problema adicional es la creciente dependencia de América Latina de sus exportaciones de recursos naturales, como se muestra en el gráfico 4 . Este hecho ha tenido en el corto plazo un efecto favorable 
para los exportadores de productos básicos de la región, gracias a un superciclo de precios altos, especialmente de productos mineroenergéticos ${ }^{4}$. Sin embargo, es muy probable que este superciclo se agote en el futuro cercano, sobre todo si la economía china, que ha impulsado el alza de los precios, entra en una fase de desaceleración más aguda que lo previsto hasta ahora.

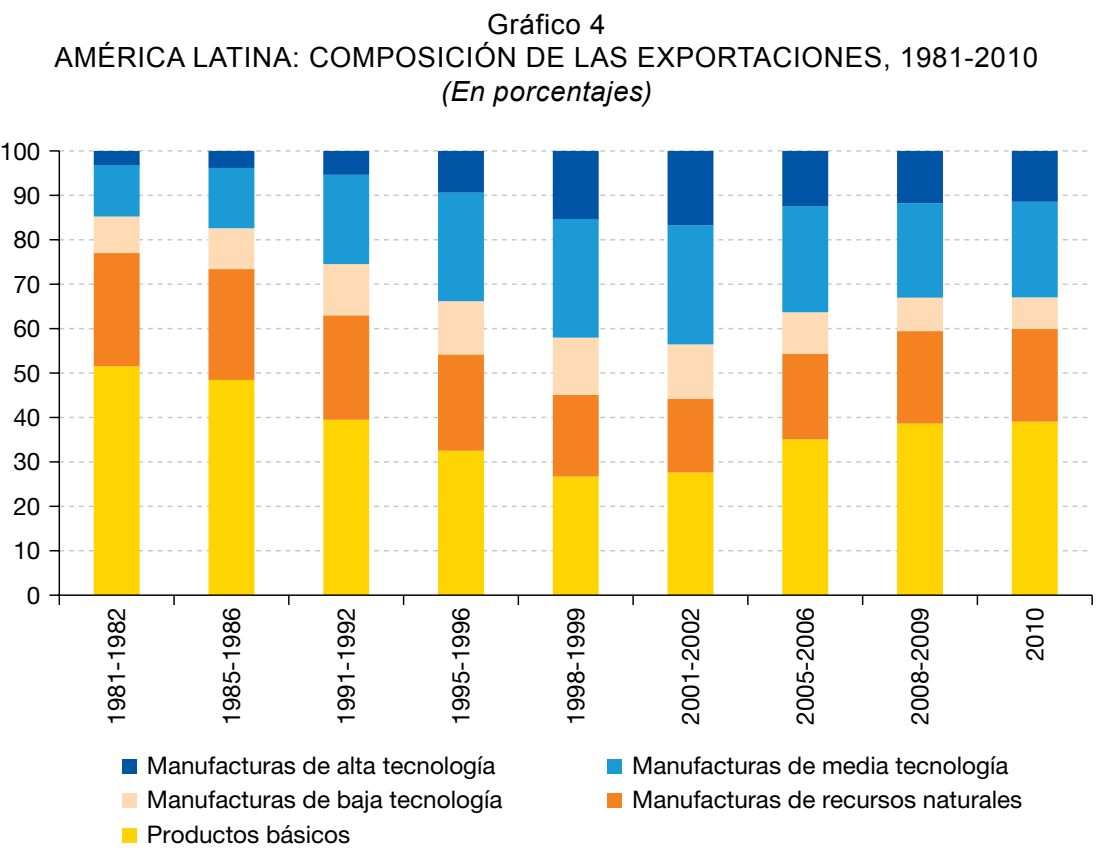

Fuente: Comisión Económica para América Latina y el Caribe (CEPAL).

¿Puede compensar la periferia de la economía mundial este escaso dinamismo del centro? La respuesta es, por una parte, positiva, ya que los países en desarrollo han superado el ritmo de crecimiento de los desarrollados en casi cinco puntos porcentuales desde $2003^{5}$. Por otra parte, el crecimiento de los países en desarrollo siguió siendo muy sensible al patrón cíclico de los desarrollados, como se manifestó en la acentuada

4 Véase al respecto Bilge Erten y José Antonio Ocampo, "Super-cycles of commodity prices since the mid-nineteenth century", DESA Working Paper, No 110, Departamento de Asuntos Económicos y Sociales de las Naciones Unidas, febrero de 2012.

5 Un 6,1\% comparado con un 1,4\%, respectivamente, en el período 2002-2012, según estimaciones de las Naciones Unidas. En 1970-2000 había sido inferior a dos puntos: un $4,7 \%$ y un $3,0 \%$, respectivamente. 
desaceleración generada por la crisis financiera mundial de 2008-2009 y nuevamente en 2012. Por otra parte, este acelerado ritmo de crecimiento del mundo en desarrollo ha recibido un fuerte impulso de la economía china, que enfrenta profundos desequilibrios estructurales, en particular una excesiva dependencia de tasas de inversión muy altas (cercanas al 50\% del PIB) y, por el contrario, bajísimas contribuciones del consumo privado (en torno al 35\% del PIB). Ello refleja un modelo de desarrollo con elevadas transferencias de los hogares a las empresas, que deben desmontarse gradualmente para dar paso a un modelo en el que el consumo privado juegue un papel más importante. Algunos analistas consideran que tal reordenamiento de los patrones de la demanda agregada no se pueden dar sino en medio de una pronunciada desaceleración de la economía de China, no al $8 \%$ previsto en la mayor parte de las previsiones actuales, sino quizás al $5 \%$ o incluso menos.

Los intercambios comerciales entre América Latina y China muestran, por otra parte, un gran desequilibrio estructural, cuya característica más destacada es la concentración de las exportaciones de nuestra región a ese país en un puñado de productos básicos (petróleo, cobre, mineral de hierro, soja y pulpa de papel) a cambio de un conjunto diversificado de productos manufactureros con creciente contenido tecnológico. Este modelo de comercio ha generado, como es obvio, un auge en muchas economías sudamericanas, pero no es el más conveniente para dinamizar la estructura productiva de la región.

Por último, cabe señalar que una de las implicaciones más importantes de estas tendencias de la economía mundial es que, en ausencia de un pronunciado dinamismo del comercio internacional, privilegian el mercado interno de los países como fuente de dinamismo relativo. En dicho sentido, podría pensarse que es una oportunidad invaluable para el mercado interno ampliado que generan los procesos de integración regional. Como lo ha señalado la CEPAL a lo largo de muchas décadas, una de las virtudes de los flujos comerciales intrarregionales es su mayor contenido de manufacturas y, en general, de manufacturas con mayor contenido tecnológico. Sin embargo, los procesos de integración sudamericanos vienen experimentado crisis profundas, que han afectado profundamente a la Comunidad Andina desde hace varios años, pero ahora se han extendido al MERCOSUR, donde las restricciones al comercio bilateral entre sus dos principales socios es una señal preocupante de la pérdida de compromiso con la integración. 


\section{Repensar la agenda del desarrollo}

Este conjunto de legados históricos y escenarios internacionales previsibles nos llaman la atención sobre la necesidad de repensar a fondo los patrones y estrategias de desarrollo latinoamericanos. Es preciso consolidar e incluso profundizar los avances, en particular en materia de estabilidad macroeconómica y reducción de los niveles de pobreza. Al mismo tiempo, es esencial analizar a fondo otros aspectos.

En materia macroeconómica, es necesario consolidar lo que ya se ha logrado en materia de reducción de la inflación, sostenibilidad fiscal y disminución del endeudamiento externo neto. Pero también es evidente que queda mucho más que hacer para reducir la histórica vulnerabilidad externa de las economías latinoamericanas. La respuesta a la crisis global de 2008-2009 fue positiva para América Latina en muchos sentidos: no hubo crisis financieras internas ni de balanza de pagos, ni ningún desborde inflacionario, y muchos países pudieron poner en marcha políticas anticíclicas, especialmente en materia monetaria y crediticia. Sin embargo, no se pudo evitar una fuerte contracción inicial del PIB regional, que de hecho fue una de las peores del mundo en desarrollo. Más aún, el auge que precedió a la crisis mundial reciente y el retorno de los capitales y de los altos precios de los productos básicos desde mediados de 2009 han demostrado que todavía queda mucho también por aprender en materia de manejo de las bonanzas, para evitar en particular la tendencia cíclica a la revaluación de las monedas (que resulta particularmente ilógica en economías con vocación exportadora), al aumento del gasto público cuando los recursos son abundantes y, aún más, al rápido crecimiento del crédito y del gasto privado que caracterizan estos períodos. 
preciso, se trata no solo de avanzar en la mejora de los indicadores de desarrollo humano y reducir la pobreza, sino de enfocar la atención en la desigualdad en sí misma. Conviene recordar, en particular, no solo que América Latina sigue siendo una de las regiones más desiguales del mundo, sino también que su nivel de desarrollo humano está inmensamente sesgado por los altos niveles de desigualdad. Ese hecho se hace evidente al mirar con atención los indicadores de desarrollo humano ajustados por desigualdad que publica regularmente el Programa de las Naciones Unidas para el Desarrollo (PNUD) desde 2010, según el cual América Latina pierde mucho más que las regiones más cercanas en niveles de desarrollo humano (las economías en transición de Europa oriental y Asia central, y los países de Asia y el Pacífico) debido a los mayores niveles de desigualdad, no solo de ingresos, sino de acceso a la educación y a la salud.

La tarea en este campo tiene tres elementos básicos. El primero es la creación de capacidades, en particular, dada la etapa actual de desarrollo de la región, en el acceso a la educación secundaria y superior y en la calidad de la educación que reciben los sectores de menores ingresos en todos los niveles. El segundo es el desarrollo de sistemas universales de protección social, cuya prioridad es, entre otras, la lucha contra la segmentación en la provisión de servicios, que implica en particular que la calidad de los servicios sea muy diferente para distintos sectores sociales. El tercero es realizar un mayor esfuerzo de redistribución fiscal, tanto por la vía del sistema tributario como del gasto público. En esta última área, cabe recordar que, aunque existen también diferencias en la distribución del ingreso antes de impuestos y gasto público, la gran diferencia en materia distributiva entre América Latina y los países desarrollados de la Organización de Cooperación y Desarrollo Económicos (OCDE) es precisamente el alcance de la redistribución que se lleva a cabo por la vía fiscal.

En todo caso, siguiendo las enseñanzas de la historia, los avances en este plano no serán duraderos si no se articulan con las necesarias transformaciones tecnológicas y productivas. El tema clave en este campo es la generación de empleos de calidad, en términos de calificación, estabilidad laboral y acceso a la protección social. Esta es una tarea en la cual existe una gran agenda pendiente, ya que desde la crisis de la deuda hasta comienzos del siglo XXI hubo un deterioro sustancial en alguno o varios de los indicadores de calidad del empleo en casi todos los países de la región, que solo ha comenzado a superarse parcialmente a partir del auge de 2003-2007. Cabe agregar que ese esfuerzo debe estar íntimamente ligado al de mejorar la estructura productiva, lo que implica que un criterio básico de la política de desarrollo productivo debe ser generar empleos de calidad. 
Los retos son incluso mayores en términos de desarrollo productivo, esencial para superar el crecimiento económico, que ha sido frustrante para muchos países latinoamericanos durante la fase de reformas de mercado, especialmente en materia de productividad. La historia económica de la región, así como las experiencias de los desarrollos productivos más exitosos de Asia oriental, indican que el objetivo de alcanzar altas tasas de crecimiento no se logrará solo con una macroeconomía sana ni con la mera especialización acorde con las ventajas comparativas estáticas. Se requieren también políticas productivas activas, un tema que fue explícitamente excluido de la agenda de los gobiernos durante la fase de reformas de mercado y en el que no se han dado pasos firmes en la dirección contraria en épocas recientes, salvo en el Brasil. Más aún, se requiere un salto en la formulación de políticas tecnológicas activas, un área en la que hubo también un déficit claro durante la fase de industrialización dirigida por el Estado. Este esfuerzo debe complementarse con la consolidación de los logros en materia educativa y la reversión de sus falencias, sobre todo en cuanto a la calidad y la articulación con las necesidades de transformación del sistema productivo.

El lento dinamismo previsible del comercio internacional hace aún más necesario repensar la tarea de mejorar la competitividad y la calidad de la canasta exportadora, así como el equilibrio entre el mercado interno y el externo. En tal sentido, podrían considerarse tres alternativas, que en realidad no son excluyentes y, por lo tanto, se pueden adoptar en combinaciones variables según el país. La primera es recuperar la atención en el mercado interno. Los efectos positivos que tienen las tendencias sociales que ha experimentado la región contribuyen a dinamizar el mercado interno (al "consumo de masas", para utilizar la terminología del ex Presidente Lula da Silva). Sin embargo, una estrategia de dicho tipo solo tiene perspectivas favorables en el Brasil y en mucho menor medida en algunos países de tamaño medio. Por ese motivo, es preferible la segunda estrategia: otorgar un peso creciente al mercado interno ampliado, revitalizando a fondo los procesos de integración. Sin embargo, para ello sería necesario superar las limitaciones políticas que han venido debilitando y, en algunos casos, francamente torpedeando los procesos de integración regional. La tercera estrategia es la diversificación exportadora, en dos sentidos diferentes: incluyendo objetivos explícitos de mejora del contenido tecnológico de la canasta exportadora, al mismo tiempo que se mantiene la atención en ampliar el mercado con las economías asiáticas dinámicas y en particular de China. Estos dos componentes son complementarios, ya que uno de los retos principales es precisamente mejorar la canasta de las exportaciones regionales hacia ese país.

En materia social, el reto principal es superar la deuda que representan los enormes niveles de desigualdad heredados. Para ser 
Este tema me lleva a las reflexiones finales, que se refieren a la planificación. En un contexto de alta incertidumbre, su función básica es contribuir a elaborar una visión y una orientación estratégicas, basadas en un análisis de los escenarios previsibles y en su impacto posible sobre los distintos países. Su propósito fundamental es ayudar a orientar las acciones de los agentes públicos y privados, algo que se vuelve aún más importante en medio de las turbulencias actuales. Por eso mismo, debe dar espacio a revisiones, en función no solo de los cambios del contexto internacional, sino también de la experiencia acumulada. Esa visión debe desarrollarse hoy a través de un proceso en el que participen distintos agentes sociales. Debe estar acompañado, además, de una capacidad de coordinar las actividades estatales orientadas a alcanzar los objetivos estratégicos y a evaluar las acciones de los organismos del Estado en tal sentido. Esas actividades, es decir, la prospectiva, la participación, la coordinación y la evaluación, constituyen lo que podríamos denominar las funciones básicas de la planificación. Todo ello debe formar parte de la tarea más amplia de fortalecer la institucionalidad estatal y, en especial, el desarrollo de un aparato administrativo capaz de ayudar a la sociedad a cumplir las funciones de provisión de bienes y servicios de interés colectivo, dentro de la particular combinación entre Estado y mercado que cada sociedad defina sobre la base de sus propios procesos democráticos.

Para lograr todo lo mencionado, la región necesita seguir contando con este exitoso Instituto cuyos 50 años celebramos hoy. 
Transcurrida la primera década del siglo XXI, los países de América Latina y el Caribe mantienen y refuerzan sus estrategias de crecimiento sostenido, inclusión y bienestar social, como hace décadas. La región ha aprendido de su propia experiencia y en ese empeño enfrenta los retos de un entorno internacional de incertidumbre $y$ volatilidad financiera.

El desarrollo latinoamericano, su historia, presente y futuro, han sido objeto de numerosos análisis, hipótesis y controversias. En este ensayo elaborado en el marco del quincuagésimo aniversario de la fundación del ILPES, José Antonio Ocampo hace un recorrido de los avatares, logros y desafíos del desarrollo de la región que, al igual que el resto del mundo, ha cambiado significativamente en las últimas cinco décadas $y$, a futuro, se prevé que las transformaciones se vuelvan aún más profundas y más frecuentes.

Aun reconociendo la resiliencia de la macroeconomía latinoamericana, puesta a prueba en la crisis actual, persisten las debilidades de los aparatos productivos y vigentes los riesgos del esquema de inserción externa, que se acentúa con la continuidad del auge de las materia primas, especialmente para Sudamérica. Permanece pues el reto de transitar hacia estadios de desarrollo en donde simultáneamente se cierren las brechas de ingreso, empleo, productividad, sociales y territoriales.

Ocampo identifica los desafíos del desarrollo de la región, y además ofrece sus reflexiones sobre los posibles caminos a seguir y los instrumentos de la política púbica para acometerlos. Todo ello, sin duda, precisa de un estado renovado, que contribuya a la revaloración de la política pública y que recupere en la planificación a un instrumento vigoroso y renovado para la materialización del desarrollo con igualdad.

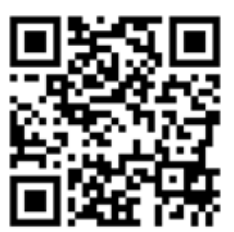

\section{www.cepal.org/ilpes}

